\title{
Layered Switch Architectures for High-Capacity Optical Transport Networks
}

\author{
Lih Y. Lin, Member, IEEE, Ezhan Karasan, Member, IEEE, and Robert W. Tkach, Senior Member, IEEE
}

\begin{abstract}
We propose and analyze layered switch architectures that possess high design flexibility, greatly reduced switch size, and high expandability. The improvement in loss and crosstalk due to the reduced switch size is also discussed. Theoretical models have been developed to compute the network blocking probability using these architectures. Low blocking probability and high network utilization are achieved because of the capability of communication between layers in adjacent switches. The results show that the proposed layered switch architectures are very attractive for high-capacity optical transport networks.
\end{abstract}

Index Terms-Blocking probability, expandability, link utilization, optical networking, switch architecture.

\section{INTRODUCTION}

$\mathbf{T}$ WE long-distance optical transport network with wavelength-division multiplexing (WDM) technology has shown a promising future for high-capacity optical fiber communications [1], [2]. In such a network, the communications between central offices are conveyed by the fiber-trunk mesh. Each central office acts as a network node, where signals from different fiber trunks and local client offices are either switched to different output fiber trunks for transmission to the next node, or dropped to the local offices.

With today's fast-growing traffic demands, the communication capacity required is increasing rapidly. Increasing the bit-rate of individual wavelength channels is one solution. This requires upgrading the bandwidth of all the network components, however, and the cost can be high. Another solution is to increase the number of wavelengths and/or the number of fibers in each fiber trunk. In such a network, an optical layer crossconnect allowing rearrangeable automated interconnection of this large number of signals will be useful. As WDM systems with tens of channels are deployed on cables with tens of fibers, crossconnects with hundreds of ports will be required. Therefore, optimal switch architecture with manageable switch size and high expandability is essential.

To avoid the formidable number of switch points a fully connected switch fabric requires, we propose here layered switch architectures that take advantage of a particular feature of any large fiber network. The nodes of the network have many signals incident on them but have relatively small

Manuscript received July 3, 1997; revised April 1998.

L. Y. Lin and R. W. Tkach are with the Lightwave Networks Research Department, AT\&T Laboratories-Research, Red Bank, NJ 07701-7033 USA (e-mail: lylin@ research.att.com).

E. Karasan was with AT\&T Labs-Research, Red Bank, NJ 07701-7033 USA. He is now with the Department of Electrical and Electronics Engineering, Bilkent University, 06533 Ankara Turkey.

Publisher Item Identifier S 0733-8716(98)05744-8.

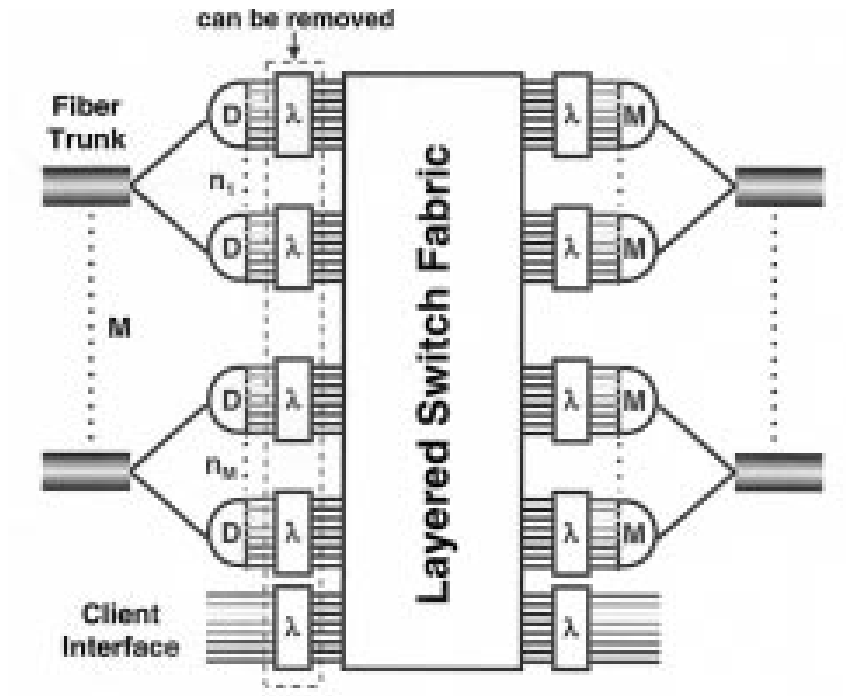

(D) : Wavelength Demultiplexer (M) : Wavelength Multiplexer

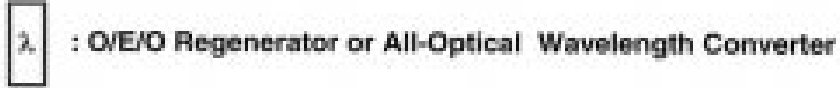

Fig. 1. The switch fabric in each network node can utilize the $\mathrm{O} / \mathrm{E} / \mathrm{O}$ regenerators or all optical wavelength converters to eliminate the wavelength dependence.

numbers of routes into them. Thus, a signal arriving at the node does not need full access to every signal path; rather, it needs access to any route leaving the node. Therefore, good performance in terms of blocking of connections can still be achieved even though the switch fabrics at individual network node are not fully connected.

Although the proposed layered architectures bear similarity to the wavelength-selective cross connect (WSXC) architecture [2]-[4], the work shown here treats the layering as a desirable feature in itself, independent of the number of wavelengths used for transmission. In addition to greatly reduced switch size, the proposed switch architectures - because of the wavelength-independence-also possess high design flexibility, high expandability, and low blocking probability.

Wavelength independence can be realized by two approaches (see Fig. 1). First, the O/E/O regenerators in the current long-haul optical transport network [1], [5] can be employed to convert the multiwavelength to a standard wavelength at the switch fabric. Second, for an all-optical transparent network, all-optical wavelength converters [6] can be incorporated. The regenerators and wavelength converters 


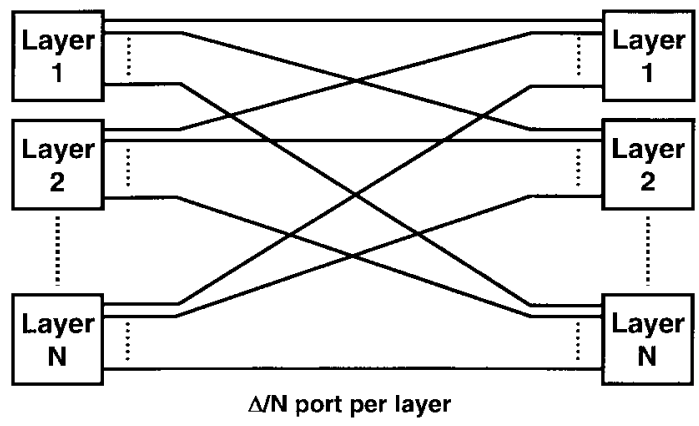

Fig. 2. Schematic drawing of the single-stage layered switch architecture. The communication between layers is achieved by cross-connecting different layers in adjacent network nodes.

before the switch fabric can be eliminated if regeneration before switching and wavelength uniformity at switching are not required. The flexibility of arbitrarily layering the switch fabric is still retained if variable-in/fixed-out wavelength conversion is utilized after switching.

In Section II, the layered switch architecture design will be described. The reduction in switch size because of the layering will also be discussed. Smaller switch scale reduces the loss and crosstalk of the switch fabric. This will be analyzed in Section III. In Section IV, the theoretical models for analyzing the blocking probability of the layered switch architectures will be presented. The advantages of the layered switch architectures in terms of reduced blocking probability and enhanced network utilization and expandability will also be demonstrated.

\section{Switch ARChitecture Design AND SWITCH SIZE ANALYSIS}

For the first approach, the switch fabric at the network node is separated evenly into $N$ layers. The schematic drawing of the switch fabric is shown in Fig. 2. Because of the wavelength independence, the communication between different layers is achieved with an "orthogonal" arrangement of the switch fabrics between adjacent network nodes, therefore, the blocking probability is reduced. Physically, the orthogonal arrangement is accomplished by crossconnecting different layers between the network nodes along the transport path. In contrast to the WSXC architecture, the number of layers does not depend on the number of wavelengths used for transmission, and high design flexibility is realized. Furthermore, the switch fabric is readily expandable by inserting more switch layers should there be increased demand for network capacity.

By layering the switch fabric, the dimensions of the switch layers can be dramatically reduced to a manageable size [7]-[9] for a high-capacity network. In the following analysis, the nonlayered, fully connected switch fabric is used as the standard for comparison and demonstration of the reduction in switch size in the layered switch architectures. It has a dimension (defined as the number of input or output ports) of

$$
\Delta=\sum_{i=1}^{M} W \cdot n_{i}+n_{c}
$$

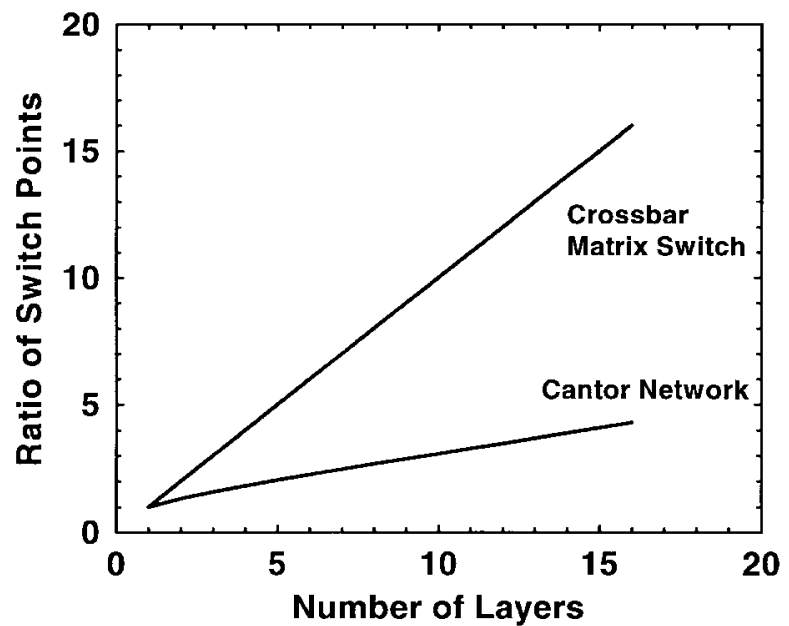

Fig. 3. The ratio of the number of switch points in the nonlayered switch fabric to that of the single-stage layered switch fabric versus the number of layers. The dimension of the switch fabric is assumed to be 256 .

where

$M$ number of fiber trunks going in/out of the switch fabric;

$W$ number of wavelengths used for transmission;

$n_{i} \quad$ number of fiber pairs (including fibers for restoration path) in each trunk;

$n_{C}$ number of local client fibers.

The number of switch points depends on the approach for constructing the switch fabric. For example, it is equal to $\Delta^{2}$ for crossbar matrix switches, and $\Delta / 2 \cdot\left(\log _{2} \Delta\right) \cdot\left(2 \log _{2} \Delta-\right.$ 1) for Cantor networks (strictly nonblocking Benes networks, ignoring the crosspoint count for the multiplexers and demultiplexers) [10], [11].

For the single-stage layered switch architecture, the switch fabric is divided into $N$ layers. Therefore, the dimension of each layer is reduced to $\Delta / N$. The number of switch points becomes $\Delta^{2} / N$ for crossbar matrix switches, and $\Delta / 2 \cdot\left(\log _{2}(\Delta / N)\right) \cdot\left(2 \log _{2}(\Delta / N)-1\right)$ for Cantor networks. To illustrate the reduction in the number of switch points by layering the switch fabric, Fig. 3 shows the ratio of the number of switch points in a fully connected switch fabric to that in a layered switch fabric versus the number of layers. The number of input/output ports $\Delta$ is assumed to be 256 . The ratio increases as the number of switch layers increases, showing the merit of arbitrarily layering the switch fabric. The effect of layering the switch fabric is more dramatic for crossbar matrix switches than for Cantor networks, because the number of switch points of Cantor networks increases logarithmically with the dimension of the switch layers. The layered switch architecture, however, has a larger influence on loss and crosstalk in Cantor networks, as will be discussed in the next section.

The blocking probability of the network traffic can be further reduced if two switches with orthogonal layering directions are cascaded in the same network node, as illustrated in Fig. 4. This also results in the uniformity of fiber interconnect in the network. The reduction in the number of switch points follows a trend similar to that in the single-stage layered switch 


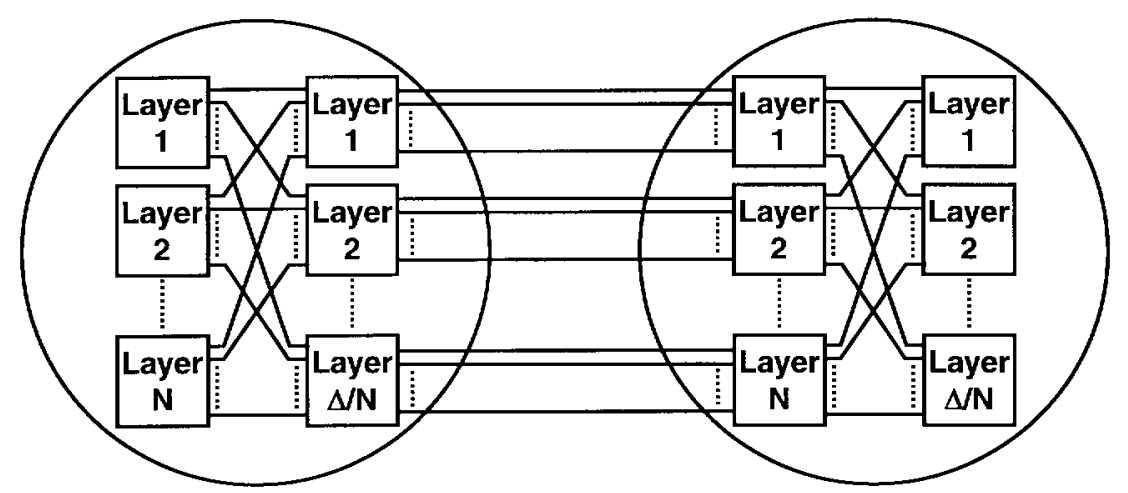

Fig. 4. Schematic drawing of the two-stage layered switch fabric. Two switches with "orthogonal" layering directions are cascaded in the same network node.

architecture. The improvement is nevertheless reduced, since both switches contribute to the switch points.

\section{Reduction IN Loss AND CROSSTALK}

In most switch fabrics, loss and crosstalk increase with the size. Therefore, by layering the switch fabric and reducing the dimension of each switch layer, its loss and crosstalk are reduced. Since most guided-wave photonics switches adopt Benes network (Cantor network) approaches, while the crossbar matrix switch is more often used by free-space fiber-optic switches (see, for example, [12] and [13]), where loss and crosstalk have insignificant dependence on the scale of the switch layers, only the Cantor network is discussed in this section.

The loss and signal-to-crosstalk ratio (SXR) in rearrangeably nonblocking Benes networks have been discussed in [14]. In a strictly nonblocking Cantor network, each input and output are connected to the corresponding input and output of $\log _{2} \Delta$ planes of Benes network switching fabrics through demultiplexers and multiplexers (passive splitters and combiners). This factor needs to be taken into consideration when calculating the loss and crosstalk of Cantor networks. The total optical loss through a Cantor network includes fiber/switchfabric coupling loss, demultiplexer loss, and losses across the switch points. It is expressed as

$$
L_{T}^{N L}(\mathrm{~dB})=2 L_{F}+L_{D}^{N L}+\left(2 \log _{2} \Delta-1\right) L_{S}
$$

for a fully connected nonlayered switch fabric, where $L_{F}$ is the fiber coupling loss, $L_{D}^{N L}=-10 \log \left(\log _{2} \Delta\right)$ is the demultiplexer loss (assuming only power splitting loss contributes), and $L_{S}$ is the loss through each switch point (assuming it is uniform across the switch fabric). For the single-stage layered switch architecture, the total optical loss in one layer becomes

$$
L_{T}^{L}(\mathrm{~dB})=2 L_{F}+L_{D}^{L}+\left(2 \log _{2}(\Delta / N)-1\right) L_{S}
$$

where the demultiplexer loss $L_{D}^{L}$ is equal to $-10 \log \left(\log _{2}(\Delta / N)\right)$.

In a fully loaded Cantor network, the signal gathers crosstalk through every switch point along its path as well as crosstalk from other planes of the Benes network through the multiplexer. Assuming uniform crosstalk through the switch fabric, the total first-order crosstalk power is

$$
P_{\text {out }}^{N L} P_{\text {in }} x_{C}\left(2 \log _{2} \Delta-1\right)+P_{\text {in }} x_{C}\left(\log _{2} \Delta-1\right)
$$

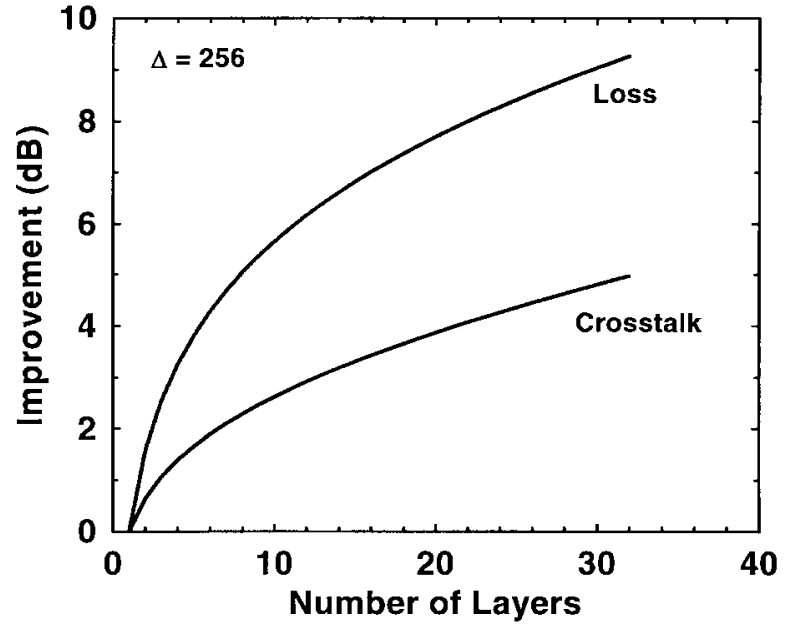

Fig. 5. Improvement in loss and crosstalk by the layered switch architecture versus the number of layers, assuming the switch fabric is constructed using the Cantor network approach. The loss through each switch point is assumed to be $0.5 \mathrm{~dB}$, and the dimension of the switch fabric is assumed to be 256 .

for nonlayered switch fabrics, where $P_{\text {in }}$ is the input optical power and $x_{C}$ is the power ratio of crosstalk at each switch point. The first term represents the crosstalk that the signal acquires through its path, and the second term is the crosstalk from other planes of the Benes network. The loss term is not considered since both signal and crosstalk paths experience the same loss and it will be canceled out in calculating the SXR. For the single-stage layered switch architecture the total first-order crosstalk power is

$$
\begin{aligned}
P_{\text {out }}^{L}= & P_{\text {in }} x_{C}\left(2 \log _{2}(\Delta / N)-1\right) \\
& +P_{\text {in }} x_{C}\left(\log _{2}(\Delta / N)-1\right) .
\end{aligned}
$$

The SXR for both nonlayered and single-stage layered switch architecture can, therefore, be calculated using (4) and (5):

$$
\begin{aligned}
\operatorname{SXR}^{N L}(\mathrm{~dB}) & =-10 \log x_{C}-10 \log \left(3 \log _{2} \Delta-2\right) \\
\operatorname{SXR}^{L}(\mathrm{~dB}) & =-10 \log x_{C}-10 \log \left(3 \log _{2}(\Delta / N)-2\right) .
\end{aligned}
$$

Fig. 5 shows the improvement in loss and crosstalk of the layered switch architecture over the nonlayered switch architecture using the Cantor network approach. The values of $L_{S}$ and $\Delta$ are assumed to be $-0.5 \mathrm{~dB}$ and 256 , respectively. The improvement increases with the number of layers. To demonstrate the crosspoint reduction advantage of layered 


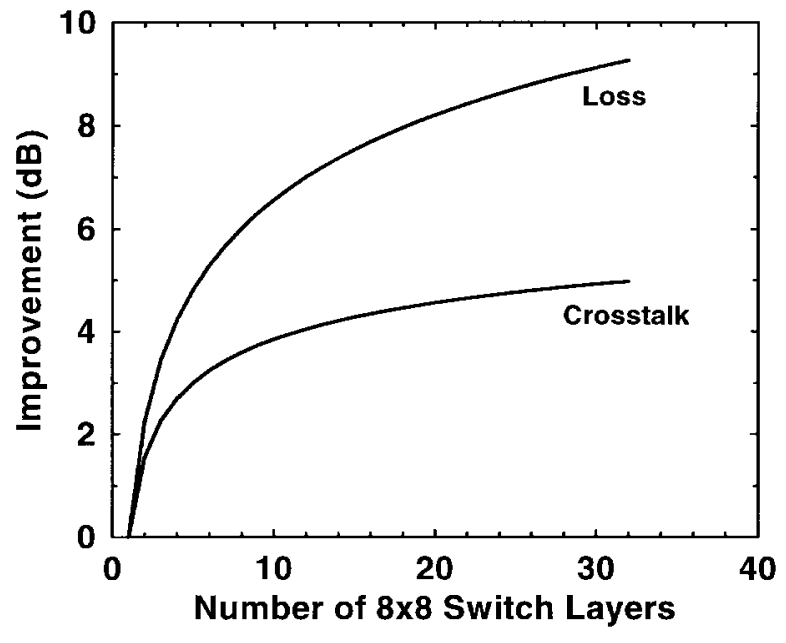

Fig. 6. Improvement in loss and crosstalk by the layered switch architecture under network capacity growth, assuming the switch fabric is constructed with Cantor network. The loss through each switch point is assumed to be $0.5 \mathrm{~dB}$.

switch architectures using the Cantor network approach under network capacity expansion, the dimension of each switch layer in the layered switch fabric is specified to be $8 \times 8$, and the loss and crosstalk versus the number of switch layers $N$ are compared with the nonlayered switch fabric which has a dimension of $8 N \times 8 N$. The results are shown in Fig. 6. The improvement in loss and crosstalk by the layered switch architectures increases with network capacity expansion. Therefore, for large-scale switch fabrics that are required in high-capacity optical transport networks, layering the switch fabric is necessary in order to reduce its loss and crosstalk as well as the switch size.

For the two-stage layered switch architecture, the analysis of loss and crosstalk follows similarly. The cascaded effect from both stages, however, needs to be considered.

\section{ANALYSIS OF BLOCKING PROBABILITY}

In this section, the blocking probability and the network utilization penalty for the proposed layered switch architectures will be compared with the wavelength-based switch architecture (WSXC) and the fully connected nonlayered switch architecture [3], [4]. Their expandability with network growth will also be analyzed.

\section{A. Fixed Number of Input/Output Ports}

The blocking probability for an optical transport path with $H$ hops and $\Delta$ input/output ports for each hop is analyzed in this section. The number of hops is equal to the number of network nodes/fiber links the signal travels through in the transport path. The single-stage layered switch architecture is analyzed first. Since each hop is not independent because of the orthogonal arrangement described in Section II, the blocking probability for each hop depends on how many layers the signal can access at each node, which is related to the blocking history of previous hops [15]. The model for simulating the blocking probability for the transport path with $H$ hops is described in the following: Let $P_{h, k}$ be the probability that $k$ layers are accessible at hop $h . P_{h, k}$ is related to the blocking history of previous hops by

$$
\begin{aligned}
P_{h, k} & =C_{k}^{N} \sum_{i=1}^{N}\left(P_{h-1, i}\left(1-\rho^{A \cdot i}\right)^{k}\left(\rho^{A \cdot i}\right)^{N-k}\right) B_{k}, \\
C_{k}^{N} & \equiv \frac{N !}{k !(N-k) !} \\
B_{k} & = \begin{cases}1, & \text { if } k \leq i \cdot \Delta / N \\
0, & \text { if } k>i \cdot \Delta / N\end{cases}
\end{aligned}
$$

where

$$
\begin{array}{ll}
N & \text { number of layers in the switch fabric; } \\
\rho & \begin{array}{l}
\text { link utilization (probability that a wavelength } \\
\text { is used on a fiber); } \\
\text { number of links connecting two layers in } \\
\text { adjacent network nodes. }
\end{array}
\end{array}
$$

The signal is blocked when there is no layer accessible at any hop along the transport path. The blocking probability for the single-stage switch architecture is therefore defined as

$$
P_{b}^{S S}=\sum_{h=1}^{H} P_{h, 0} .
$$

By employing the initial condition

$$
P_{0, k}= \begin{cases}1, & \text { if } k=N \\ 0, & \text { if } k \neq N\end{cases}
$$

the blocking probability $P_{b}^{S S}$ can be obtained.

The algorithm for analyzing the blocking probability for the two-stage layered switch architecture is analogous to that for the single-stage architecture, with two differences: 1 ) the signal on one layer has access to all the outputs on different layers, therefore, $A \cdot i$ in (8) is now replaced by $\Delta / N$ and 2) the effective link utilization $\rho_{\mathrm{eff}, k}$ for the two-stage architecture needs to be modified - a link is not accessible if either it is utilized or it is not utilized but the cross-connect of the switch fabric is arranged such that the signal cannot reach the unutilized link. If the signal can access $k$ input layers, the effective utilization $\rho_{\mathrm{eff}, k}$ is expressed as

$$
\rho_{\mathrm{eff}, k}= \begin{cases}\rho, & \text { if } k=N \\ \rho+(1-\rho)\left(\left(\frac{m-A}{m}\right)^{2}\right)^{k}, & \text { if } k \neq N\end{cases}
$$

where $m=\Delta / N$ is the number of input/output ports for each layer and $A$ means the number of paths through which the signal can access a particular output layer and has the same expression as in the previous definition. The blocking probability for the two-stage switch architecture $P_{b}^{T S}$ is now given by

$$
\begin{aligned}
& P_{b}^{T S}=\sum_{h=1}^{H} P_{h, 0} \\
& P_{h, k}=C_{k}^{N} \sum_{i=1}^{N}\left(P_{h-1, i}\left(1-\left(\rho_{\mathrm{eff}, i}\right)^{m}\right)^{k}\left(\left(\rho_{\mathrm{eff}, i}\right)^{m}\right)^{N-k}\right) B_{k} .
\end{aligned}
$$

It has been shown that taking link correlation into consideration instead of the link independence assumption reduces the 


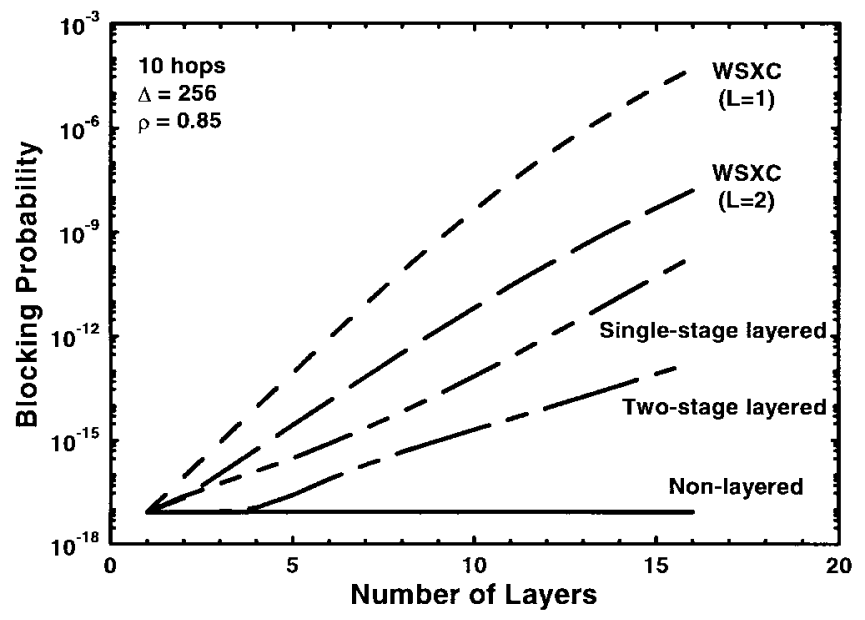

Fig. 7. Blocking probability versus the number of layers for different switch architectures.

blocking probability substantially for the WSXC architecture [3], [4]. This dependence is absorbed by the interference length parameter $(L)$ defined in [4]. $L$ is the average number of links shared by two intersecting paths; hence $L=1$ corresponds to the case with independent link loads. The interference length depends on the topology of the network and the routing algorithm used [16]. Fig. 7 shows the blocking probability versus the number of layers for the WSXC for interference length $L=1,2$ single-stage layered, two-stage layered, and nonlayered switch architectures along a transport path with ten hops. The values of $\Delta$ and $\rho$ are assumed to be 256 and 0.85 , respectively, in the calculation. For the WSXC, the number of layers is equal to the number of wavelengths. The communication between different wavelengths/layers is prohibited in the WSXC, therefore it has the highest blocking probability. Because of the wavelength independence and the ability to communicate between layers, the single-stage layered switch architecture has substantially lower blocking probability than the WSXC, while the advantage of switch-size reduction remains unchanged.

Although WSXC with long interference length has lower blocking probability, an average interference length $(L=2)$ in the transport network still results in a higher blocking probability than the single-stage layered switch architecture. The blocking probability performance of the two-stage architecture approaches that of the nonlayered switch fabric. A three-stage architecture (Clos network) achieves the performance of the nonlayered switch architecture [15].

An alternative characterization of the performance of these switch architectures in a network is the "utilization penalty," which indicates how much more network investment is required to reach a given performance for each switch architecture. It is defined as the percentage of additional channels in the fiber trunks required to achieve a specific blocking probability when compared to the fully connected, nonlayered switch architecture. Fig. 8 shows the utilization penalty for different switch architectures assuming the blocking probability equals $10^{-10}$. The layered architectures have much lower utilization penalties compared to WSXC.

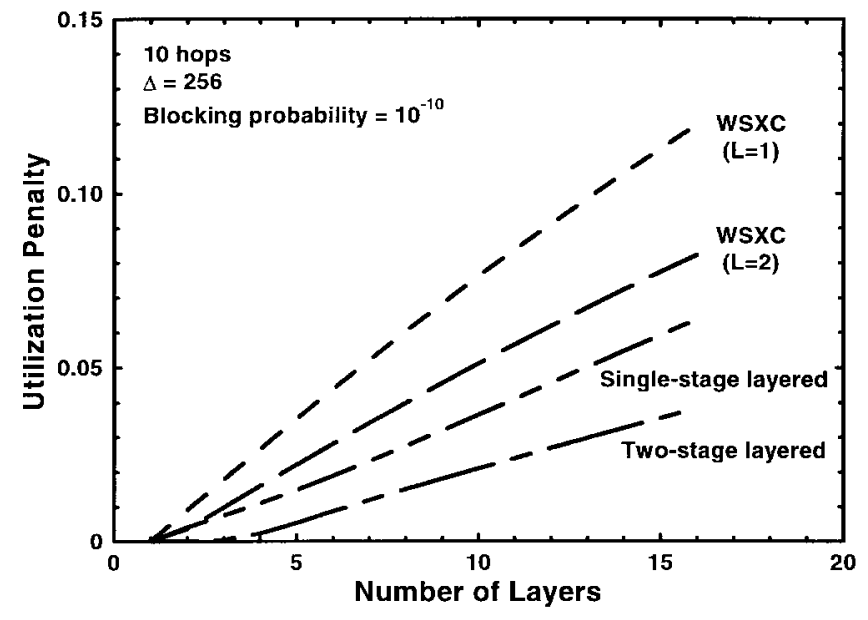

Fig. 8. Utilization penalty (compared to the nonlayered switch architecture) versus the number of layers for different switch architectures.

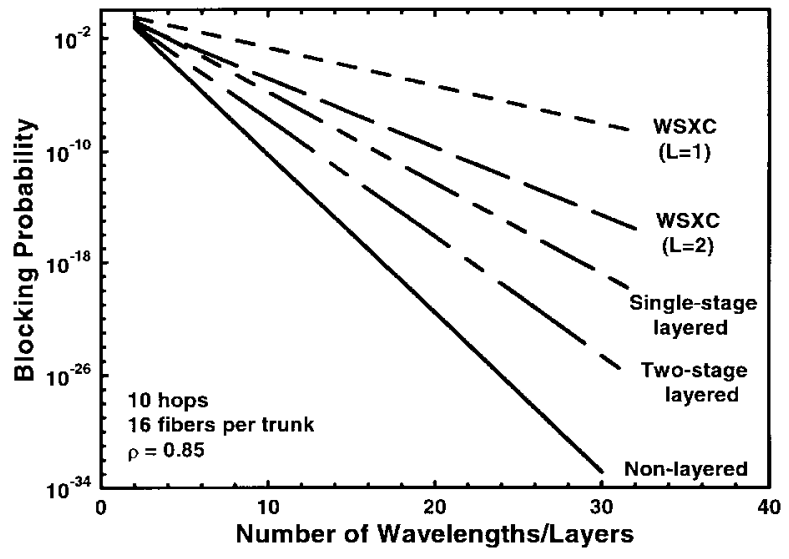

Fig. 9. Blocking probability versus the number of wavelengths for different switch architectures. The number of input/output ports in each switch layer is 16 . The blocking probability for the nonlayered switch architecture is also plotted for comparison. It has a dimension of ( $16 \times$ number of wavelengths).

\section{B. Growth with Network Capacity}

To reduce the cost of upgrading network capacity, a switch architecture should be designed such that when there is expansion in network traffic, the switch fabric in each network node is also growable by adding additional switch components instead of requiring total replacement of the switch fabric. The nonlayered switch architecture is not suitable if the network capacity expansion is achieved by increasing the number of wavelengths and/or fibers in the fiber trunk, since the number of its input/output ports is fixed. The WSXC architecture is modular if capacity increases by adding wavelengths, but not if the number of fibers increases. Therefore, we compare the expandability of WSXC and the two-layered architectures assuming the network capacity expansion is realized by increasing the number of wavelengths.

The blocking probability under network capacity expansion is shown in Fig. 9. The link utilization $\rho$ is assumed to be 0.85. The number of hops in the transport path is equal to ten, and the number of fibers in each trunk is 16 . The dimension of each layer is also chosen to be 16 so that the number of wavelengths is equal to the number of layers for all 
three architectures. For comparison, the blocking probability of a nonlayered switch architecture with a dimension of (16 $\times$ number of wavelengths) is also plotted, although this kind of switch architecture is nonexpandable. As the number of wavelengths increases, the blocking probability decreases because more channels are available to the signal. The proposed layered switch architectures have much lower blocking probability compared to the WSXC architecture. Therefore, layered switch architectures are nicely scaleable in response to rapidly growing bandwidth demand in highcapacity optical fiber transport networks.

The simulation results show that the proposed layered switch architectures achieve network performance close to a fully connected switch architecture. On the other hand, it also increases complexity of network control and management. Nevertheless, as high-port-count switch fabrics are essential for the emerging high-capacity mesh-based optical networks and most switching technologies cannot meet the stringent demands, these approaches provide a means of realizing such networks if the network control and management algorithm can be designed and planned appropriately.

\section{CONCLUSION}

In summary, we have proposed and analyzed the singleand two-stage layered switch architectures. These architectures do not depend on the number of wavelengths and, therefore, possess high design flexibility. By layering the switch fabric, the dimension of each switch layer can be greatly reduced to a manageable size, and the switch fabric is readily scaleable by inserting more switch layers. The loss and crosstalk of the switch fabric are decreased because of the smaller switch sizes. Theoretical models have been developed for analyzing the blocking probability. The blocking probability is substantially reduced relative to the wavelength-based layering architecture. Thus, the link utilization is enhanced because of the capability of communication between layers. Simulation results show that the proposed layered switch architectures can satisfy the capacity demands of the network while remaining manageable in size. Therefore, they are very attractive for realizing optimal and cost-effective switch architecture for high-capacity optical transport networks.

\section{REFERENCES}

[1] E. L. Goldstein, J. A. Nagel, J. L. Strand, and R. W. Tkach, "Multi wavelength opaque optical crossconnect networks," Lightwave Mag., pp. 92-98, Feb. 1998.

[2] R. E. Wagner, R. C. Alferness, A. A. M. Saleh, and M. S. Goodman, "MONET: Multiwavelength optical networking," IEEE J. Lightwave Technol., vol. 14, pp. 1349-1355, June 1996.

[3] G. Jeong and E. Ayanoglu, "Comparison of wavelength-interchanging and wavelength-selective cross-connects in multiwavelength all-optical networks," in IEEE INFOCOM'96, San Francisco, CA, Mar. 1996, pp. $156-163$.

[4] R. A. Barry and P. A. Humblet, "Models of blocking probability in all-optical networks with and without wavelength changers," IEEE $J$. Select. Areas Commun., vol. 14, no. 5, pp. 858-867, 1996.

[5] K. Bala, R. R. Cordell, and E. L. Goldstein, "The case for opaque multiwavelength optical networks," in IEEE LEOS Summer Topical Meeting on Technologies for a Global Information Infrastructure, Keystone, CO, July 1995 , pp. 58-59.

[6] L. H. Spiekman, U. Koren, M. D. Chien, B. I. Miller, J. M. Wiesenfeld, and J. S. Perino, "All-optical Mach-Zehnder wavelength converter with monolithically integrated DFB probe source," IEEE Photon. Technol. Lett., vol. 9, pp. 1349-1351, Oct. 1997.

[7] E. J. Murphy, T. O. Murphy, A. F. Ambrose, R. W. Irvin, B. H. Lee, P Peng, G. W. Richards, and A. Yorinks, "16 $\times 16$ strictly nonblocking guided-wave optical switching system," IEEE J. Lightwave Technol., vol. 14, pp. 352-358, Mar. 1996.

[8] C. Burke, M. Fujiwara, M. Yamaguchi, H. Nishimoto, and H. Honmou, "128 line photonic switching system using $\mathrm{LiNbO}_{3}$ switch matrices and semiconductor traveling wave amplifiers," IEEE J. Lightwave Technol., vol. 10, pp. 610-615, 1992.

[9] L. Y. Lin, E. L. Goldstein, J. M. Simmons, and R. W. Tkach, "Highdensity micromachined polygon optical crossconnects exploiting network connection symmetry," IEEE Photon. Technol. Lett., to be published.

[10] V. E. Benes, Mathematical Theory of Connecting Networks and Telephone Traffic. New York: Academic, 1965.

[11] D. Cantor, "On nonblocking switching networks," Networks, vol. 1, Dec. 1971.

[12] L. Y. Lin, E. L. Goldstein, and R. W. Tkach, "Free-space micromachined optical switches with submillisecond switching time for large-scale optical crossconnects," IEEE Photon. Technol. Lett., vol. 10, pp. 525-527, Apr. 1998.

[13] H. Toshiyoshi and H. Fujita, "Electrostatic micro torsion mirrors for an optical switch matrix," IEEE J. Microelectromech. Syst., vol. 5, pp. 231-237, Dec. 1996.

[14] H. S. Hinton, J. R. Erickson, T. J. Cloonan, and G. W. Richards, "Spacedivision switching," in Photonics in Switching, vol. II, J. E. Midwinter, Ed. New York: Academic, 1993.

[15] J. Y. Hui, Switching and Traffic Theory for Integrated Broadband Network. New York: Kluweric, 1990.

[16] E. Karasan and E. Ayanoglu, "Effects of wavelength routing and selection algorithms on wavelength conversion gain in WDM optical networks," IEEE/ACM Trans. Networking, vol. 6, pp. 186-196, Apr. 1998.

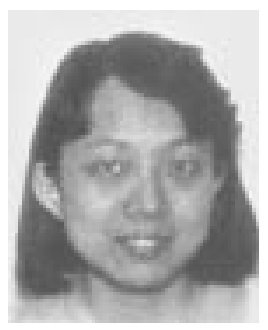

Lih Y. Lin (S'93-M'96) received the M.S. and $\mathrm{Ph} . \mathrm{D}$. degrees in electrical engineering from the University of California at Los Angeles, in 1993 and 1996, respectively.

She has been a Senior Technical Staff Member at the Lightwave Networks Research Department, AT\&T Laboratories-Research, Red Bank, NJ, since 1996. Her research has been in the areas of micromachine switching technologies and switching architectures for high-capacity optical networks and four-wave-mixing wavelength conversion for WDM crossconnects. She has more than 70 publications in technical journals and conferences.

Dr. Lin is a Member of OSA and the Photonics Society of ChineseAmericans. She was awarded the Dr. Bor-Uei Chen's Memorial Scholarship from the Photonics Society of Chinese-Americans in 1995. In 1997, she was awarded the Outstanding Doctor of Philosophy, the highest honor accorded to Ph.D. graduates of the School of Engineering and Applied Science, the University of California at Los Angeles.

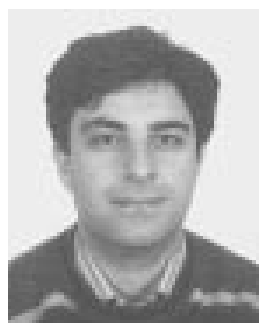

Ezhan Karasan (M'95) received the B.S. degree from Middle East Technical University, Ankara, Turkey, and the Ph.D. degree from Rutgers University, Piscataway, NJ, all in electrical engineering, in 1987, 1990, and 1995, respectively.

During 1995-1996, he was a Researcher at Bell Labs, Holmdel, NJ. From 1996 to 1998, he was a Technical Staff Member in the Lightwave Networks Research Department at AT\&T Labs-Research, Red Bank, NJ. He is currently an Assistant Professor in the Department of Electrical and Electronics Engineering, Bilkent University, Ankara, Turkey. He has worked on the Long Distance Architecture Task of the Multiwavelength Optical Networking (MONET) project, which is sponsored by DARPA. His current research interests are in the design and performance analysis of high-speed network architectures and multimedia systems.

Dr. Karasan was the recipient of a fellowship from NATO Science Scholarship Program between 1991 and 1994. 


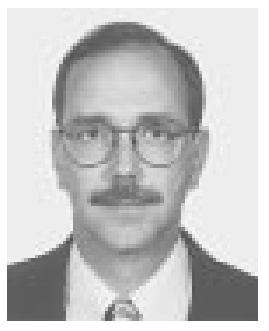

Robert W. Tkach (M'84-SM'98) was born in Lorain, OH, in 1954. He received the Ph.D. degree in physics from Cornell University, Ithaca, NY, in 1982.

From 1982 to 1984 , he was a Postdoctoral Research Associate at Cornell University. He has been at AT\&T Bell Laboratories, Red Bank, NJ, since 1984, investigating various aspects of lightwave communications systems including semiconductor lasers, optical networks, and nonlinear optical effects in fibers. He is currently head of the Lightwave Networks Research Department, AT\&T Laboratories. His research has been in the areas of demonstration of ultrahigh capacity transmission systems based on wavelength division multiplexing and on the application of optical networking in long-haul transport networks.

Dr. Tkach is an AT\&T Fellow and a Fellow of the Optical Society of America. 